\title{
Study Protocol Version Study Population Description
}

National Cancer Institute

\section{Source}

National Cancer Institute. Study Protocol Version Study Population Description. NCI

Thesaurus. Code C94290.

The textual representation of the subject characteristics, including inclusion and exclusion criteria and describes the population for which the study may be generalized. 